\title{
The Design of Protected Agriculture Monitoring Information System Based on GIS- A Case Study of Qiqihar
}

\author{
Cheng Wang ${ }^{1}$ and Chan-juan Pang ${ }^{2}$ \\ ${ }^{1,2}$ Information Management and Information System Department, School of \\ Economics and Management of Qiqihar University, Qiqihar, 161006, China \\ 1wang-cheng@sohu.com, ${ }^{2}$ sjkzy@sina.com
}

\begin{abstract}
According to the current situation and requirements of Qiqihar protected agriculture, this paper proposes a GIS-based construction scheme of protected agriculture monitoring information system of Qiqihar, which is based on the business requirement of decision-making of Qiqihar Municipal Agricultural Economic Committee, and elaborates the detailed requirement analysis and the system design process. The scheme proposed by this paper can provide services in scientific decision-making, technique and market information for Qiqihar Municipal Agricultural Economic Committee. It can improve the level of management and the ability of decision-making of Qiqihar protected agriculture effectively.
\end{abstract}

Keywords: GIS, protected agriculture, monitoring information system

\section{Introduction}

Protected agriculture is the only way to make agriculture free from the control of nature and make the agriculture become modern factory, environmentally safe and no poison by using man-made facilities [1]. The protected agriculture is high input and high output, capital and technique and labor-intensive industry. The informatization of protected agricultural is an important content in the development of modern agriculture, in increasing farmers' income and in the construction of a new socialist countryside [2].The construction of Chinese protected agriculture began from the later 1980s. After decades of development, it has made remarkable achievements, but it is long behind the country whose protected agriculture develops better. The disadvantages are mainly in the following aspects. Firstly, the level of facilities is low, poor in the ability of resisting natural disasters. Secondly, the level of mechanization is low. The production is still human-based with great labor intensity and low labor productivity. Thirdly, the technique of protected agriculture is not suitable, not standard and low-tech. It is lack of quantitative indicators and quite blind in technical application and production. So, it is quite difficult to achieve the efficiency of protected agriculture and larger-scale productions. Lastly, cultivated technique is backward and the management is not efficient [3]. The city of Qiqihar is a major agricultural city in the west of Heilongjiang province. In recent years, it has a rapid development in the construction of protected agriculture. The productions are extending from vegetables to flowers and fruit trees, becoming the most dynamic and advantaged new industries. The position and role of protected agriculture is more and more important in the city's agriculture and rurally economic development. It has achieved high economic efficiency. In 2011, the area of protected agriculture added 1.89 million acres, totally to 9.53 million acres, ranking first in total area of greenhouse in the country. However, lacking of effective monitoring means, the 
protected agriculture of Qiqihar faces certain problems in the developing of protected agriculture. On the one hand, the cropping structure is similar, leading to fierce competition in price and difficult to increase the income of farmers. The same kind of product produced in different areas at the same time influence the price of each other, turning out disorderly competition. This makes the price of some vegetables decline greatly, and brings certain loss to farmers. On the other hand, the management is traditional and the monitoring is lag behind. Because of this, it is not efficient to find and correct problems. Now it is difficult to adapt to the increasingly complex situation and needs only relying on the traditional methods and means. It is an inevitable choice that rely on computer, network, GIS and other information technologies to steadily manage the information about the construction and structural adjustment of protected agriculture, requirements and supplies in market and so on to improve the scale and efficiency of protected agriculture simultaneously in Qiqihar[4].

Based on reviewing the current situation and requirements of protected agriculture in Qiqihar and researching the business requirement of decision-making of Qiqihar's Rural Economic Committee, we proposed a construction scheme of protected agriculture monitoring information system of Qiqihar based on GIS. It provides services in scientific decision-making, technique and market information for all links of protected agriculture in Qiqihar. It can improves the level of management and the ability of decision-making of protected agriculture in Qiqihar, achieve the goal of using high technology to transform traditional industries and promote the informatization of agriculture in Qiqihar.

\section{Requirements Analysis}

Requirements analysis is one of the key points of success in system construction. In the constructing process of GIS-based protected agricultural monitoring information system of Qiqihar City, we will stick to principles of demand-led and prominent key points, and consider the development of protected agriculture and the actual needs of information construction. Requirements analysis mainly includes five aspects, which are user requirements, business requirements, functional requirements, data requirements and performance requirements.

\subsection{User Requirements Analysis}

Focus on the purposes and requirements of the GIS-based protected agricultural monitoring information system of Qiqihar city, the users of the system are mainly divided into internal users and external users. The details of users are shown in Figure 1.

Internal users mainly refer to the staff of Qiqihar Municipal Agricultural Economic Committee, including administrators, the leadership, the subdivisions and institutions of Agricultural Economic Committee, as well as local agricultural sectors. Since the system is deployed in the Information Center of the Agricultural Economic Committee, the administrators of the Information Center will have all the rights of the monitoring system. The leadership can access to the decision support system and know well about the industrial layout and developing trend of protected agriculture in order to timely and correctly make decisions. Each department and institution of Qiqihar Municipal Agricultural Economic Committee will add their own information and those submitted by the local sectors of protected agriculture into the system. Each town and county submits their statistical information through the data submitting system. At last that information will be submitted to the information center by the agricultural departments of counties. 


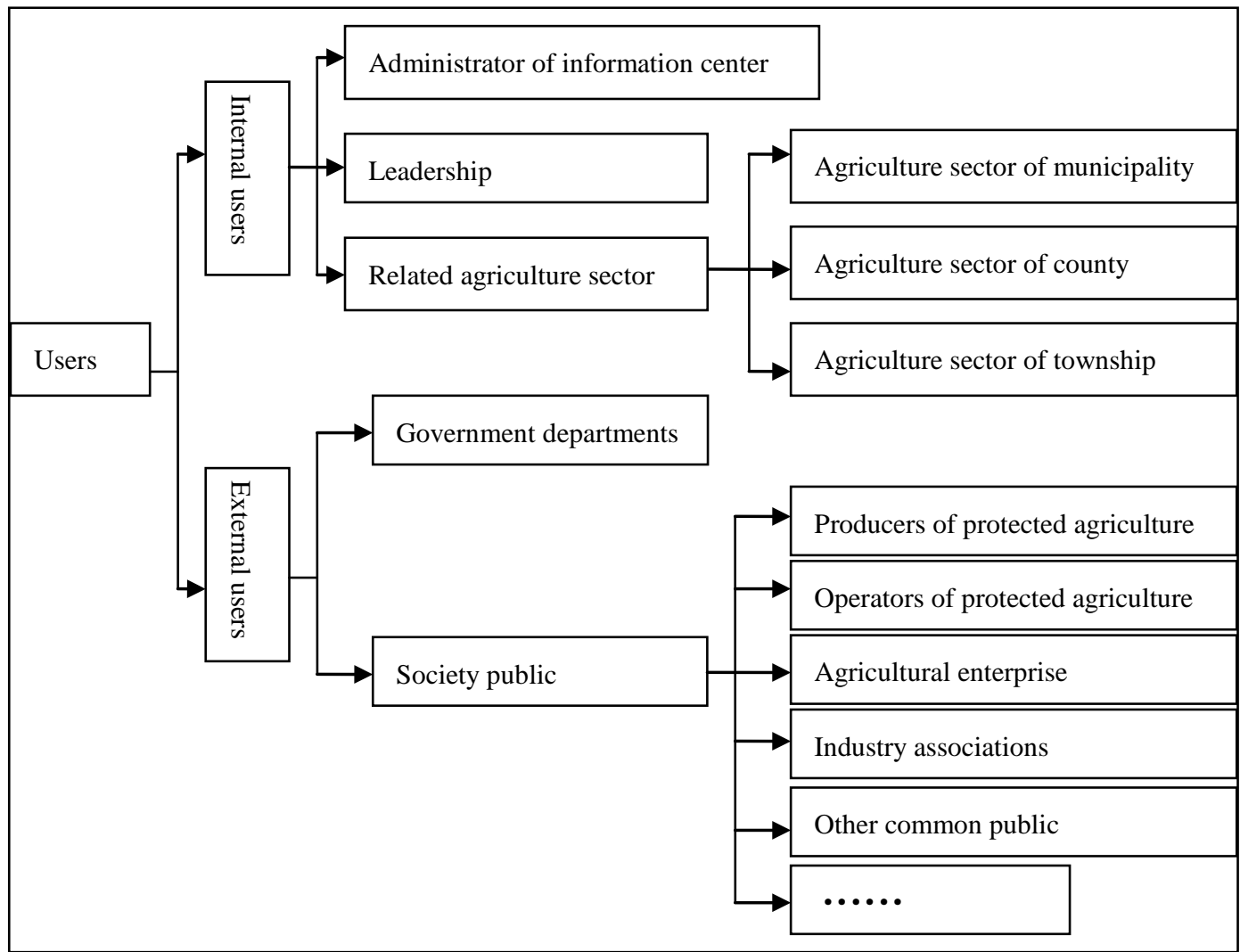

Figure 1. User Information

External users can be divided into two groups. One group is the government sectors except the Agricultural Economic Committee of Qiqihar, including all levels and relevant departments. The other group is the public, including producers and operators of protected agriculture, agricultural enterprises, industry associations, general public and so on. They can use a geographic information system to browse related information, etc.

\subsection{Business requirements analysis}

Protected agricultural layout planning and macro-decision-making are complex and systematic works concerning microscopic structure and macroscopic systems [5]. There are large amounts of data needed to be analyzed and solved timely. If the work is done by man, it will be a heavy workload and a very slowly progress. Besides, it's easy to make mistakes and can't promise to provide decision-making services to related sectors timely and effectively. Using analysis and decision support system combined with geographic information systems not only can make up for the disadvantage of manual handling, but also can directly and accurately reflect the relevant information on electronic map, such as the layout of protected agriculture, crops, scale, contacts, way of contact. It is useful to further work. The flow of business requirements is shown in Figure 2. 


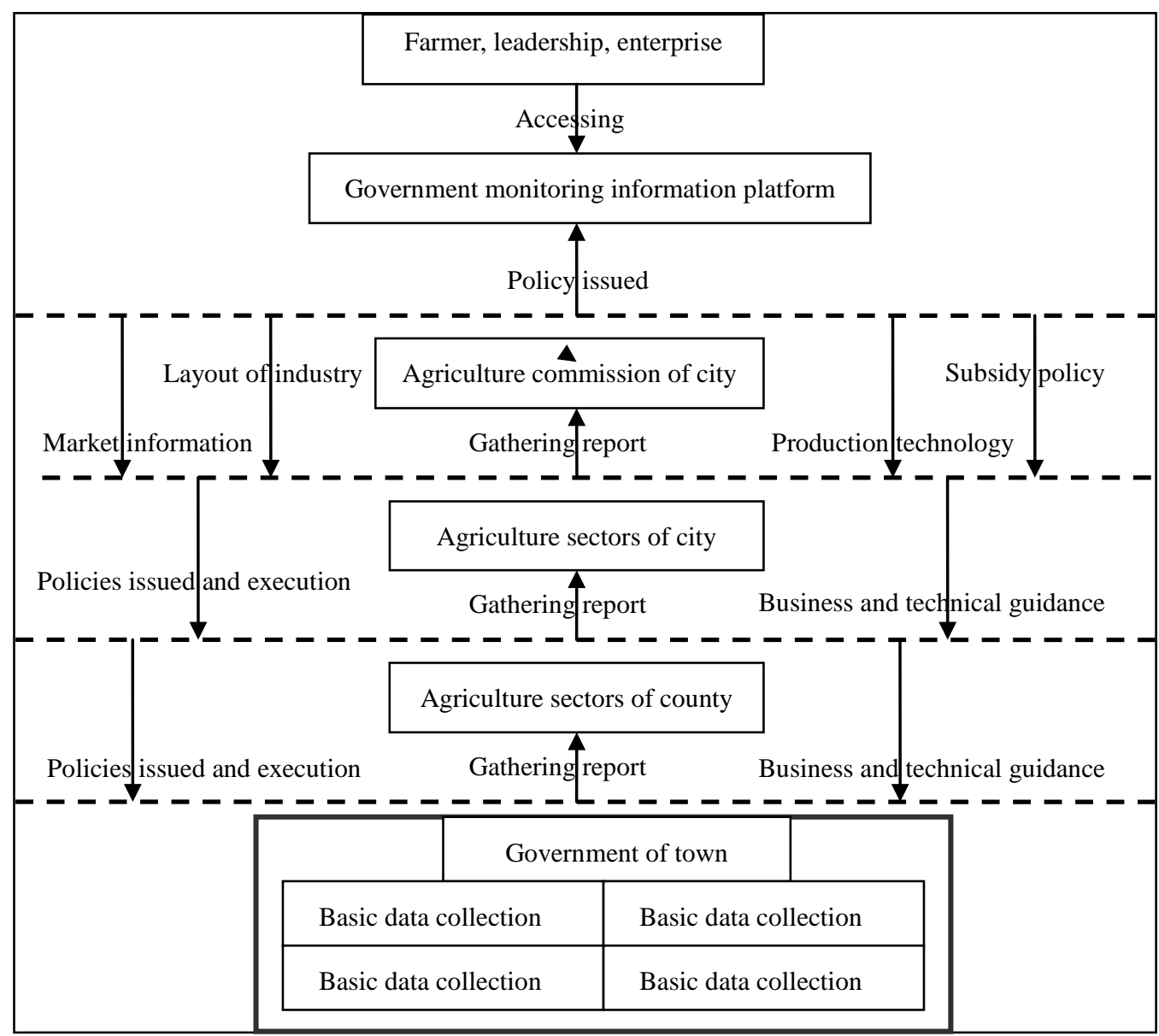

Figure 2. The Flow of Business Requirements

\subsection{Functional Requirements Analysis}

According to the actual fact of protected agriculture in Qiqihar, the functional requirement of GIS-based protected agriculture monitoring information system includes three aspects, which turn out to be production management of protected agriculture, data submit and data analysis.

2.3.1. Production Management of Protected Agriculture: It is used to collect the information of protected agriculture of the whole city and to show the specific location of farms, planting areas and big growers. When clicking on a farm on the map, it will link to its home page which shows its planted crops, scale, contacts, contact methods, amounts of subsidies and so on. When the map is narrow to county and the mouse is over a township, it will show the area of every crop and its estimated output, also show the contacts and contact methods of the agriculture sectors. When the map is narrow to Qiqihar city, it will show the information of its protected agriculture. At the same time, it can achieve the operation of selecting data, such as selecting and highlighting the areas on the map that have a larger planting area than a certain value. Beside, one can select the specific technological service sectors and their information, such as address and contact methods. 
2.3.2. Data Submit Function: Passing first-hand data through the Internet timely and accurately, such as text messages and image information, the information will be real-time acquisition and pass by process. Thus, real-time monitoring and distributed processing of the city's protected agricultural information will come true. Through efficient combination of privilege management and function of data submit, we can ensure the one-to-one relationship between the submitted data and the processing staff, so as to ensure the accuracy of uploading data.

2.3.3. Data Analysis Function: With the powerful spatial analyzing function of GIS (overlap analysis, buffer analysis, aggregation cluster analysis), it can estimate the distribution of human resources and planting resources, the fertility of soil, the diffusing speed of disasters, the light and sunshine, and so on. According to the collected and submitted data, the state of distribution can be predicted. Besides, combined with specific geographical environment, distribution of existing facilities, potential demands and other factors, it can provide scientific basis for the locating of new bases and service agencies, through analyzing by the analytical methods of overlap analysis, buffer analysis and density analysis and so on. After data analysis and processing, the results will be shown in the form of chart or text, such as the planted scale trend graph, historical curve analysis.

\subsection{Data Requirements Analysis}

Based on the requirements of system design, we divide monitoring data of protected agriculture of Qiqihar into basic data, geospatial data and management data. Basic data includes detailed information of planted bases, farms and technological service sectors of protected agriculture. Geospatial data includes the 1:10000 administrative divisions GIS data of Qiqihar City. Management data includes information of system administrators and operation logs, and other data for system managing and operating [6].

\subsection{Performance Requirements Analysis}

Performance of the system is the chief factor in constructing a system. In the process of constructing the system, performance requirement of the system is analyzed from the following aspects: Scalability, efficiency, reliability, operability, security and maintainability.

2.5.1. Scalability: The system is based on components, fully considering its scalability. It is provided with a relatively perfect framework which is easy to expand in function and application. Mean-while, its data structure is clear and can meet the needs of constructing business systems or other professional application systems in the future. The database capacity of the system can reach to TB level.

2.5.2. Efficiency: The system uses a load balancing strategy to solve the problem of high-load access and large number of concurrent requests. The system can support the access of more than 100 concurrent users. Those can ensure that the system run efficiently and stably.

2.5.3. Reliability: The system should run normally and stably to ensure the integrity of the data. The system should supply a $7 * 24$ hours continuous operation and the annual accumulative failure time should less than 24 hours. At last, the system should have a strong recovery capability against disasters, and promise the average fixed time is less than 24 hours. 
2.5.4. Operability: Every operation of the system must be as simple as possible for the people to operate simply. Functions of the system must achieve the need of business. Further confirmation is needed for the destructive operation of data, meanwhile could restore the data to previous operation.

2.5.5. Security: Construction of the system should fully consider the security requirements for the project. A well network information security system includes facility security, network security, database security, application security, management security and so on.

The system is rich in contents and has many users, so that it is necessary to distribute operating rights to the system users in order to achieve the functions of all aspects. For that, set up the system administrator who can add, delete, modify the internal users, assign users' rights and define their identity. More over the system has a feature of security tips. When the users operate incorrectly leading to data modified and serious consequences of the network running, the system will turn out security tips itself. Then the operation can't continue except approved by multiple grade. When maps, graphics or data are maintained, it will not disturb the normal work of the system. So in this process, it needn't to stop the system. The system has a perfect data backup function. It not only can make timing backup for the system data, but also can make backup for the specified tables or all tables in the database according to requirement. This can avoid the failure problems of system caused by data destroyed. Moreover, the recovery of backup data won't affect the stability and normal use of the system [7].

2.5.6. Maintainability: We can use the management module of the system to manage and maintain logs, data backup and so on. Applications and managements of the system must be manageable and maintainable. The maintainability is necessary for the system to run continuously.

\section{System Design}

\subsection{Goals of Design}

The general goal of the system is to construct a monitoring geographic information system of protected agriculture of Qiqihar. This system is an integrated system, a combination of all kinds of mathematical methods, basic database of protected agriculture and geographic information system. Based on the current situation and requirements of protected agriculture as well as the business requirements of Municipal Agricultural Economic Committee, we will effectively improve the service system and information level of the protected agriculture of Qiqihar. The system will provide all links of protected agriculture in Qiqihar with services in scientific decision-making, technique and market information. The detailed designed goals of the system are the following five goals.

Firstly, store and manage all kinds of data related to protected agriculture, such as agricultural production data, conventional maps, electronic maps, MSS data, TM data, GPS data, and make the heterogeneous data to be isomorphic according to application requirements. Secondly, based on basic information database and kinds of mathematical methods, perform multivariate statistical analysis of construction and species-divided production of protected agriculture. Moreover, generate the raster data of subsystem correspondingly. Thirdly, closely combine the functions of database management system, geographic information system and mathematical models. Accomplish all functions of the 
system, such as image transmitting, image scaling, image roaming, layers management, visual selecting, classified statistics, spatial analysis, multivariate statistical analysis, decision aids, image output, digital map output, data update. Fourthly, according to the output information of models, evaluate and analyze the construction of protected agriculture comprehensively and visually from multiple angles and levels. Then accomplish the government's supervision and management of protected agriculture as well as the sharing and co-constructing of information resource of protected agriculture. Fifthly, accomplish a three grade networks covering city, county, township. The users of villages, towns and agricultural organizations can submit and access information.

\subsection{Logical Framework of the System}

According to the designed goal of system and the requirements of users in different levels, the system is divided into six front operation modules (Sequential diagram module, original index analysis module, index analysis module, decision analysis module, database table selecting, plate module of electronic map) and three background supporting modules (data supporting, data management, mathematical methods). The front operation modules and the background supporting modules compose the whole system that has functions to accomplish all kinds of integrated applications [8-10]. The overall framework of the system is shown in Figure 3.

The Figure 3 contains components of data layer and that of application layer, including content management, data integration, data selecting and application integration. That form the full integration and sharing of information and services. Based on GIS platform, the system releases and manages the GIS data through the network. It uses distributed computing technology based on internet and component design structure. And it can support the application system integration of large and complex networks which are crossing the region and network [11].

\subsection{Functional Structure of the System}

As aids of the management of protected agriculture, the protected agriculture monitoring information system of Qiqihar city provides the decision-makers with valid records. The records are about production data of protected agriculture as well as natural and social conditions related to protected agriculture based on location. Through the system it is available to form various types of statistical charts, to improve the speed and accuracy of production and emergent decisions of protected agriculture. Through its characteristics that the information is transformed fast and timely, the quality of management is greatly improved as well as that of the implementation, control and coordination of plans.

According to the functional requirement, the system is designed into three modules, electronic map browsing, comprehensive management module, decision supporting module. Functions and ways of turning out are shown in Figure 4.

3.3.1. Browse of Electronic Map: The universal operation of electronic map provides usual operational functions of GIS map, such as display, browse and roaming of the map, enlarging or narrowing of the map, measuring the distance between specific locations or area of them on the map, highlight of a specific area on the map, printing, hawk eyes and layers control. Through the browsing capability, the selected information will be shown directly in graph, and we can browse the map from different angles and enlarge or narrow it as we like. 


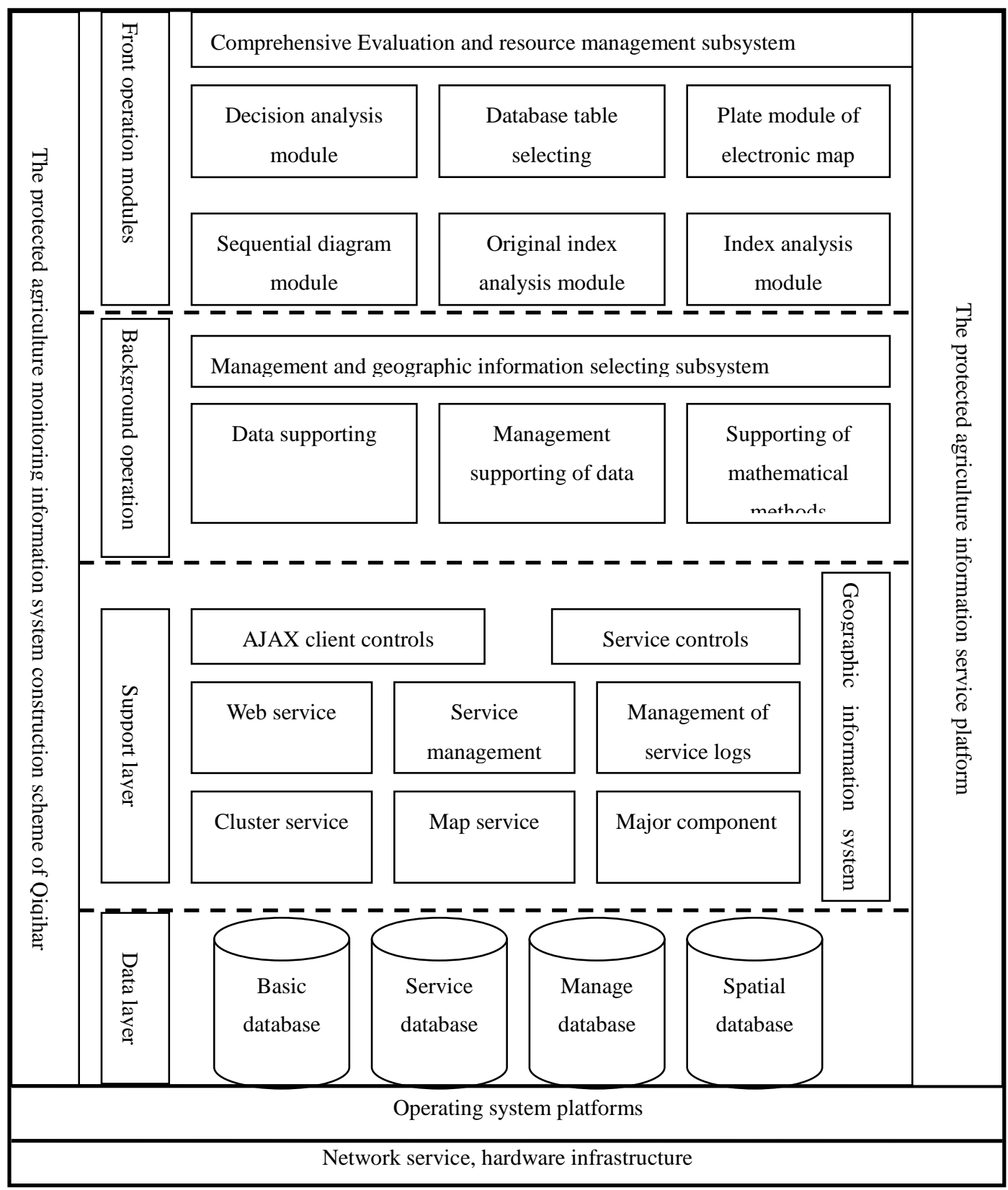

Figure 3. Overall Framework of the System

3.3.2. Comprehensive Management Module: Comprehensive management module is divided into two subsystems. One manages the system attributes. The other performs geographic information selecting. Through this module, two functions can be obtained. First, users' information and relevant business data can be managed. Second, layout of protected agriculture, scale of planting, kinds of crops and any other relevant information can be selected.

Subsystem of attributes management includes three functions, management of users and logs, management of selected output and management of business data. Management of users 
and logs includes management module of users and function module of logs. The management module of users is mainly operated by the administrator. Its operations include editing users, assigning the rights and password of users. Function module of logs can record the operating information of every user. The administrator can know when, which computer, which name the user log in the system, and which data they edit.

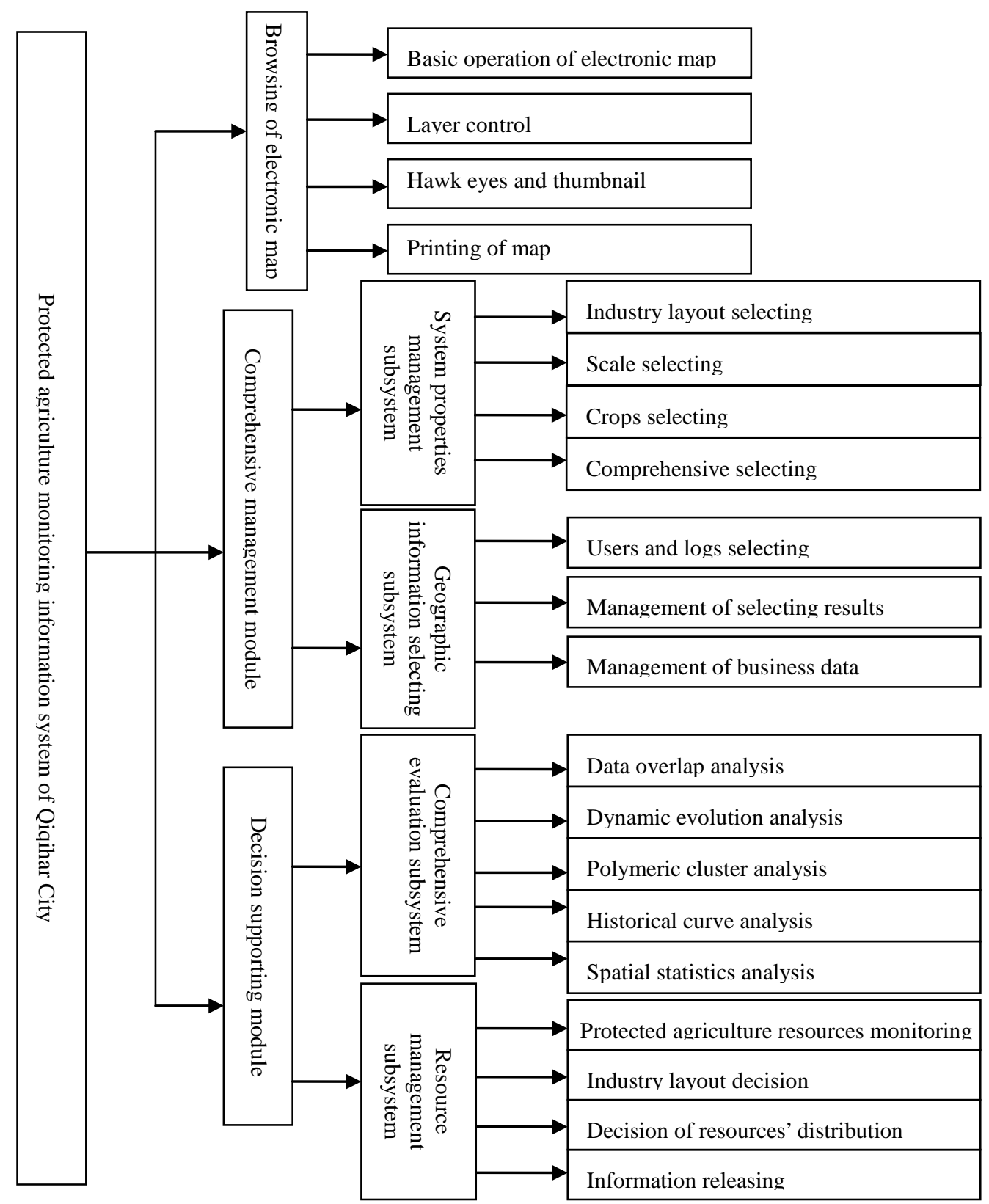

Figure 4. Structure of the System Function

The management of selected output includes the following details. (1) Buffer selected. It can select certain or all elements from the layers to create a regional layer which is within certain distance from the selected elements. One can specify the needed distance in the 
attribute table of the layer. (2) Select attribute data from graphic data. Through geometric figure, such as rectangle, arbitrary polygon, select an area on the graph and highlight it with a color, then the system will show its attribute information one by one. (3) Raster layer selected. The system can select raster cells and save them as a new raster layer according to any logical expressions given by users. (4) Select from attribute database to electronic map. The system can select the specified spatial location and highlight it with certain color on the map, according to the related information (single or a combination of logical expressions) given by users. (5) Select database from electronic map. It not only can accomplish one-to-one selecting, but also can accomplish one-to-many selecting. The former refers to that one element on the map relate to one record in the attribute database. The latter refers to that one element on the map relate to several records in the attribute database. (6) Classification of elements and manufacture of thematic maps. According to the different attributes, the elements are endowed with different symbols, especially endowed with color changed, or size changed or density changed symbols following the changing value of attributes. Or endow the elements with symbols according to their corresponding practical matters. (7) Improve the typesetting function in the output of graph-text reports. The reports can be loaded with maps, text, tables, charts and other information carriers. Moreover, the position and appearance of the information carriers could be adjusted as one like. The reports can be printed directly. (8) Export images in common formats. The system can export the reports which are map-view or typographical well in common formats, such as JPG, BMP.

Based on township and according to the regional distribution, management of business data updates the production information about planted bases, farms and planters, etc. Agricultural sectors of county use the platform to summarize the regional distributing information of township within their jurisdictions. Then the regions summarize the information of county and at last the Municipal Agricultural Economic Committee. Thus, it can reflect the distribution, scale and other information of protected agriculture of township and county in the city more comprehensively, timely, and correctly. Every grassroots sector maintains the basic data of protected agriculture through the module.

Through the subsystem of geographic information selecting, the users can select the distribution, scale and other information of the protected agriculture, such as protected agriculture's distribution, planted scale, planted crops and overall information. All kinds of information will be shown in thematic graph. The selecting subsystem of geographic information systems will facilitate our selecting for information. Selecting of protected agriculture's distribution can show bases, farms and top growers of protected agriculture. Each selected data can be shown in different thematic graph. Selecting of planted scale can tell the developing scale of protected agriculture from levels of city, county or township, and show the results on the electric map. Selecting of planted crops can select the current situation of protected agriculture in different regions according to the kinds of planted crops, and show the data on the electric map. Selecting of overall information can tell the developing scale of protected agriculture, affected farmers, the amount of subsidy and other overall information and show it on the electric maps.

3.3.3. Decision Supporting Module: Decision supporting module is divided into two subsystems, overall evaluation subsystem and resource management subsystem. Combining specifically analytic method with mathematical model, the module can assign the resources of protected agriculture, so it can provide the decision-makers with scientific basis of decision-making. Besides, that releasing agriculture-related information to the external is convenient for the farmers and other users to get the information of market and the policy and 
planning of government in time. It also can increase the quality of production and the income of farmers.

The overall evaluation subsystem can analyze the original data and show the results in text or graph, such as overlying analytical method, aggregation and cluster analysis, dynamic evolution analysis, historical trend analysis, spatial statistical analysis. Overlying analytical method can show the data submitted by towns or county on the electric maps, then overlie the regional electric maps, at last obtain the electric map which reflects all the information of protected agriculture of the whole city. Aggregation and cluster analysis can aggregate all kind of selected information that is the same or duplicate, according to certain knowledge rules, then cluster the aggregated information, at last get the accurate, clear and detailed classified information. It is useful to classify and store the data. Dynamic evolution analysis can display the evolution of protected agriculture dynamically, according to time, region, the weather and man-made conditions. Thus we can know the evolutionary process better. That is useful for the further forecast of the development trend. Historical trend analysis can show the developing process of protected agriculture in forms of curve by analyzing the data. Spatial statistical analysis refers to analyze the thematic data of geography in the database statistically.

Subsystem of resource management can monitor the resource of protected agriculture. It can make decisions of the industrial layout and the distribution of resource as well as release information and other operations. It achieves the unified management and distribution of agricultural resources through this module. Firstly, according to the analysis above, the system can give a graph of developing trend of protected agriculture and corresponding data tables. That can help the government to make decisions accurately, timely and efficiently. Secondly, according to the information of different kinds and scales of protected agriculture, the system can specify the scope of planted areas of specific crops and mark it on the electric map. Then it is convenient for the government to schedule the layout of protected agriculture scientifically. Lastly, based on the system, the Municipal Agricultural Economic Committee releases recent information of protected agriculture to the whole city regularly, such as developing situation, the newest industrial layout, requirements' changing in the market. That will help farmers to choose crop and scale according to the market information and the policy and planning of the government. It can avoid the structural similarity of crops and avoid the breaking from the demand of market. Also, it can reduce the trading difficulties, the price competition and the phenomenon that inexpensive paddy damaging farmers.

\subsection{GIS Platform Selection}

The GIS platform is a basic supporting platform to the system. In order to well achieve the functions needed by the project, the following capacity is needed: (1) Multiple types and large amount of data supporting. It should be able to input varieties of spatial data, including EOO, MIF, DGN, DXF and so on, and can support varieties of data, such as vector, images, DEM. Meanwhile, it should access large space data quickly and support the mixed display and overlying analysis of varieties of data. (2) Varieties of spatial data, including vector, images, DEM, network, etc, can be stored in the database according to the object-relational data model, and can be fast access. (3) It can support the generating method of pure components and varieties of development languages. It can build system interface as like, and can access all kinds of information of objects from the component interface. (4) Application server, processing tools of desktop data, desktop component developing platform and the whole series of mobile GIS development platform are available to meet the need of expansion of system function and scale. (5)It should be able to support the newest development technology 
of web service, so as to satisfy the requirement of interconnection and interoperability of systems in the future. At the same time, efficiently decrease the costs of development and maintenance.

\section{Conclusions}

In this paper, considering the specific business of Municipal Agricultural Economic Committee, we proposed a GIS-based system construction scheme based on the current situation and requirements of Qiqihar protected agriculture. Through its successful implementation, scientific monitoring and layout, guiding of scientific decision-making and timely macro-control can be provided for protected agricultural in Qiqihar. It will be convenient for the government to adjust the industrial structure of the regional agriculture efficiently and timely. It will help to overcome market failures and decrease the destruction of ecological environment caused by the traditional growth mode of agriculture. It can improve the level, quality and added-value of agricultural productions and save a lot of resource at the same.

\section{References}

[1] Anonymous, “The Future of Protected Agriculture”, American Vegetable Grower, vol. 1, (2012).

[2] G. Feng, Y. Li, L. Shang-qiong, X. Qing-xiang and Y. Li-jie, "Status and development trend of facility agriculture in foreign countries”, Journal of Zhejiang Forestry College, vol. 2, (2009).

[3] Z. Dewen, C. Yongsheng and C. Sanliu, "Questions and strategy of development of facility agriculture in China”, Agric Equip Technol., vol. 1, no. 33, (2007).

[4] A. Jiahui and C. Tingting, "The Analysis of Construction of Protected Agriculture Monitoring Information System Platform in Heilongjiang Province”, J. Forward Position, vol. 12, (2012).

[5] D. Encang, F. Lihong, L. Baoqian, Z. Weili and Z. Yijun, "The Current Situation and Developing Trend of Protected Agriculture”, J. Countryside Agriculture Farmers, vol. 3, (2005).

[6] L. Ping-ping, "Put current situation of agriculture and its development trends in perspective", Agriculture Equipment \& Technology, vol. 5, (2010).

[7] W. Mao-hua, "Development of precision agriculture and innovation of engineering technologies”, Journal of Agricultural Engineering, vol. 2, (2009).

[8] L. Pei-wen, "Discuss agricultural Informational and construction of the socialist new countryside", J. Agriculture network information, vol. 4, (2007).

[9] Arora, "Study on Technologies for Information Monitoring and Quick Response of Agricultural Focuses and Significant Events”, J. Agricultural Sciences in China, vol. 5, (2010).

[10] C. Serodio, J. B. Cunha, R. Morais, C. A. Couto and J. L. Monteiro, “A networked platform for agricultural management systems”, J. Computers Electron, vol. 4, (2001).

[11] H. X. Ren and L. W. Gao, "Research Progress on Expert System Technology and its Application in Plant Protection”, China Plant Protection, vol. 11, (2007).

\section{Authors}

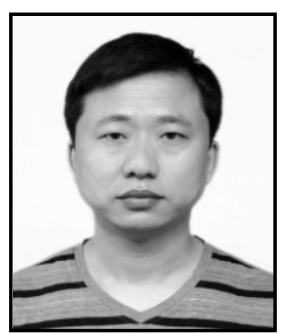

Wang Cheng, he received his M.Sc. in Management (2002) from Harbin Engineering University. Now he is associate professor at information management and information system department, Qiqihar University. Since 2012 he is Vice-President of school of economics and management. His current research interests include different aspects of information management and information system. 


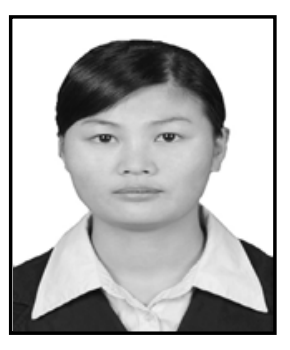

Pang Chan-juan, She received her B. Admin. in Information Management and Information System (2009) from Central South University. Now she is a postgraduate student of Qiqihar University. Her current research interests include different aspects of technology and economics. 
International Journal of $\mathrm{u}$ - and e- Service, Science and Technology Vol.6, No.5 (2013) 